\title{
Compton Scattering on Light Nuclei
}

\author{
Deepshikha Shukla ${ }^{1, a}$ \\ The University of North Carolina, Chapel Hill, North Carolina, USA.
}

\begin{abstract}
Compton scattering on light nuclei $(A=2,3)$ has emerged as an effective avenue to search for signatures of neutron polarizabilities, both spin-independent and spin-dependent ones. In this discussion I will focus on the theoretical aspect of Compton scattering on light nuclei; giving first a brief overview and therafter concentrating on our Compton scattering calculations based on Chiral effective theory at energies of the order of pion mass. These elastic $\gamma \mathrm{d}$ and $\gamma \mathrm{He}-3$ calculations include nucleons, pions as the basic degrees of freedom. I will also discuss $\gamma \mathrm{d}$ results where the $\Delta$-isobar has been included explicitly. Our results on unpolarized and polarization observables suggest that a combination of experiments and further theoretical efforts will provide an extraction of the neutron polarizabilities.
\end{abstract}

\section{Introduction}

Neutron structure is governed by strong-interation dynamics and hence its electromagnetic properties encode information that contribute to our understanding of Quantum ChromoDynamics (QCD). For example, an early success of the $S U(3)$ quark picture was its prediction of magnetic moments, $\mu$, for the neutron and other strongly-interacting particles (hadrons). Magnetic moments are a first-order response to an applied magnetic field. In this discussion however, the focus is on electromagnetic polarizabilities. The two most basic polarizabilities are the electric and magnetic ones, $\alpha_{E 1}$ and $\beta_{M 1}$, which quantify the second-order response of an object to electric or magnetic field (that produces an induced dipole moment). The Hamiltonian for a neutral particle (in this case, a neutron) in applied electric and magnetic fields, $\mathbf{E}$ and $\mathbf{B}$, is then:

$$
H=-\mu \cdot \mathbf{B}-2 \pi\left[\alpha_{E 1}(\omega) \mathbf{E}^{2}+\beta_{M 2}(\omega) \mathbf{B}^{2}\right] .
$$

Eq. (1) contains terms that are quadratic in the applied electromagnetic field. If we now consider the derivatives (first-order) of the applied fields, four new structures appear which are second order in $\mathbf{E}$ and $\mathbf{B}$ [1] and are explicitly dependent on the intrinsic spin of the object. They are:

$$
\begin{aligned}
& -2 \pi\left[\gamma_{E 1 E 1} \sigma \cdot \mathbf{E} \times \dot{\mathbf{E}}+\gamma_{M 1 M 1} \sigma \cdot \mathbf{B} \times \dot{\mathbf{B}}\right. \\
& \left.-2 \gamma_{M 1 E 2} \sigma_{i} E_{i j} B_{j}+2 \gamma_{E 1 M 2} \sigma_{i} B_{i j} E_{j}\right]
\end{aligned}
$$

with $T_{i j}:=\frac{1}{2}\left(\delta_{i} T_{j}+\delta_{j} T_{i}\right)$. The coefficients $\gamma_{\ldots .} \mathrm{s}$ are the socalled "spin polarizabilities". Eqs. (1) and (2) encode the multipole parameterizations of the polarizabilities and any other representation of the polarizabilities (for instance, spin polarizabilities $\gamma_{1}-\gamma_{4}$ ) can be expressed as linear combinations of these polarizabilities. This discussion intends

\footnotetext{
a e-mail: dshukla@physics.unc.edu
}

to show that for the neutron, these six polarizabilities can be extracted from Compton scattering on on the deuteron and ${ }^{3} \mathrm{He}$.

Polarizabilities such as those in Eqs. (1) and (2) can be accessed in Compton scattering because the effective Hamiltonian yields a nucleon Compton scattering amplitude of the form:

$$
T_{\gamma N}=\sum_{i=1 \ldots 6} A_{i}(\omega, \theta) t_{i}
$$

Here $t_{1}-t_{6}$ [2] are invariants constructed out of the photon momenta and polarization vectors $\left(\hat{\epsilon}\right.$ and $\left.\hat{\epsilon}^{\prime}\right) . t_{1}$ and $t_{2}$ contain terms that are nucleon-spin independent whereas $t_{3}-t_{6}$ include nucleon spin. The $A_{i}$ 's are Compton structure functions; expanding these $A_{i}$ around $\omega=0$ illustrates the how the polarizabilities affect the Compton amplitude. $\alpha \beta$ enter at $O\left(\omega^{2}\right)$ whereas the spin-polarizabilities enter at $O\left(\omega^{3}\right)$. For the proton, the Thomson term, $-\frac{Q^{3}}{M} \hat{\epsilon}^{\prime} \cdot \hat{\epsilon}$ ensures a larger cross section (compared to the neutron) and from low-energy Compton scattering measurements $\alpha^{(p)}$ and $\beta^{(p)}$ can be extracted. A combined analysis of the differential cross-sections (dcs) of a number of $\gamma \mathrm{p}$ experiments over the past decade [3] yields the PDG values:

$$
\begin{aligned}
& \alpha_{p}=(12.0 \pm 0.6) \times 10^{-4} \mathrm{fm}^{3}, \\
& \beta_{p}=(1.9 \pm 0.5) \times 10^{-4} \mathrm{fm}^{3} .
\end{aligned}
$$

Similar extractions for the neutron is not possible because

- the neutron Thomson term is absent and,

- there are no free-neutron targets as neutrons are very short-lived (lifetime $\sim 880$ secs.).

Of all the possible ways to access neutron polarizabilities (that includes scattering neutrons off a heavy nucleus such as lead), Compton scattering on light nuclei has emerged as likely candidates that would enable the extraction of the 
six neutron polarizabilities. For instance, the latest global analysis of the 28 points for deuteron Compton scattering gave

$$
\begin{aligned}
& \alpha^{s}=11.3 \pm 0.7_{\text {stat }} \pm 0.6_{\text {Baldin }} \pm 1_{\text {th }} \\
& \beta^{s}=3.2 \mp 0.7_{\text {stat }} \pm 0.6_{\text {Baldin }} \pm 1_{\text {th }}
\end{aligned}
$$

for the iso-scalar nucleon polarizabilities $[4,5]$ with the Baldin sum rule $\bar{\alpha}^{(s)}+\bar{\beta}^{(s)}=14.5 \pm 0.6$ as constraint. The situation for the neutron spin polarizabilities is much worse and the only data available are for the forward and the backward spin polarizabilities, which are linear combinations of the four spin polarizabilities. The neutron backward spin polarizability was determined to be

$$
\begin{aligned}
\gamma_{\pi} & =-\gamma_{E 1 E 1}-\gamma_{E 1 M 2}+\gamma_{M 1 E 2}+\gamma_{M 1 M 1} \\
& =(58.6 \pm 4.0) \times 10^{-4} \mathrm{fm}^{4},
\end{aligned}
$$

from quasi-free Compton scattering on the deuteron [6]. The $\chi \mathrm{PT}$ prediction for $\gamma_{\pi}$ is $57.4 \times 10^{-4} \mathrm{fm}^{4}$ [7]. The forward spin polarizability, $\gamma_{0}$, is related to energy-weighted integrals of the difference in the helicity-dependent photoreaction cross-sections $\left(\sigma_{1 / 2}-\sigma_{3 / 2}\right)$. Using the optical theorem one can derive the following sum rule $[2,8]$ :

$$
\begin{aligned}
\gamma_{0} & =-\gamma_{E 1 E 1}-\gamma_{E 1 M 2}-\gamma_{M 1 E 2}-\gamma_{M 1 M 1} \\
& =\frac{1}{4 \pi^{2}} \int_{\omega_{t h}}^{\infty} \frac{\sigma_{1 / 2}-\sigma_{3 / 2}}{\omega^{3}} \mathrm{~d} \omega,
\end{aligned}
$$

where $\omega_{t h}$ is the pion-production threshold. The following results on $\gamma_{0}$ were obtained using the VPI-FA93 multipole analysis [9] to calculate the integral on the RHS of Eq. (7):

$$
\gamma_{0} \simeq-0.38 \times 10^{-4} \mathrm{fm}^{4} .
$$

The $\chi \mathrm{PT}$ prediction for $\gamma_{0}$ is consistent with zero [7].

New data for unpolarized deuteron Compton scattering from MAXlab is being analysed [10], and an experiment at $\mathrm{HI} \gamma \mathrm{S}$ is approved. Additionally, the fact that a polarized ${ }^{3} \mathrm{He}$ nucleus behaves as an effective neutron [11] has generated interest in ${ }^{3} \mathrm{He}$ Compton scattering. The first elastic $\gamma^{3} \mathrm{He}$ calculations were reported in Ref. [12-14] and preparations are underway for a proposed experiment at HI $\gamma$ S. Currently, a concerted effort is underway to reduce the theory-error using higher orders in the chiral counting [15]. The goal is a comprehensive approach to Compton scattering in the proton $[16,17]$, deuteron $[4,5,16,18$ $20]$ and ${ }^{3} \mathrm{He}[12-14]$ in $\chi$ EFT from zero energy to beyond the pion-production threshold. These proceedings give a quick overview of the current investigations to help extract the neutron polarizabilities from polarized and unpolarized deuteron and ${ }^{3} \mathrm{He}$ Compton scattering.

\section{Compton scattering off a nucleon}

In order to calculate the Compton amplitude (3) at energies of the order of the pion mass, we employ Heavy Baryon Chiral Perturbation Theory ( $\mathrm{HB} \chi \mathrm{PT}$ ) with pions and nucleons as effective degrees of freedom [2]. The expansion parameter in this formulation is $Q=\left(\frac{p, m_{\pi}}{M, \Lambda_{\chi}}\right)$, where $p$ is a small momentum usually comparable to the pion mass, $m_{\pi} ; M$ is the nucleon mass and $\Lambda_{\chi}$ is the scale of chiral symmetry breaking. Fig. 1 shows the leading and the nextto-leading order contributions in this framework. At this order the theory is parameter-free and the polarizabilities are then predictions of the theory. The nucleon Compton

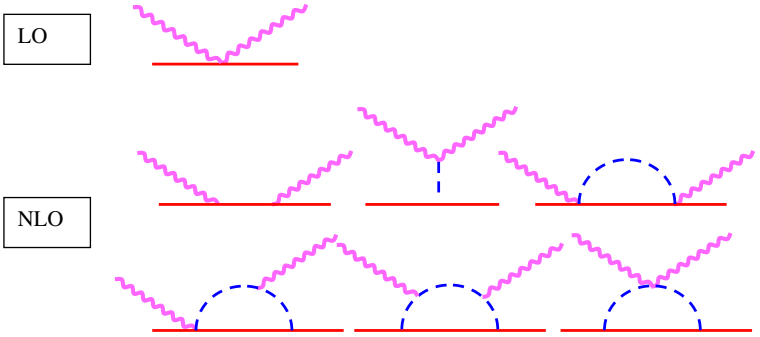

Fig. 1. The LO and NLO contributions to nucleon Compton scattering. The wiggly lines are photons, dashed ones are pions and the solid ones are nucleons. Permutations and crossed diagrams not shown.

amplitude at NNLO has also been calculated [21], however for the purpose of the results reported in these proceedings they are immaterial.

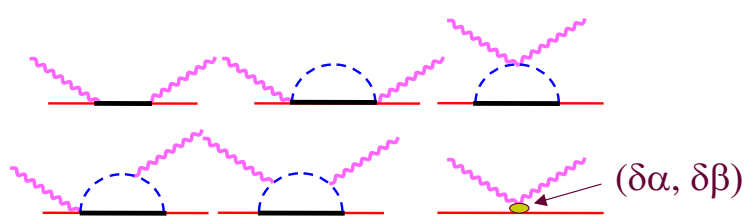

Fig. 2. The $\Delta$ contributions to nucleon Compton scattering up to NLO. The thick solid ones are depict the intermediate $\Delta$-isobar.

The $\Delta$-isobar can be included explicitly in the calculation and the results reported in these proceedings employ the "Small Scale Expansion" (SSE) scheme [22]. In this scheme the expansion parameter, $\varepsilon$ denotes either a small momentum, the pion mass, or the mass difference $\Delta_{0}$ between the real part of the $\Delta$ mass and the nucleon mass, i.e. $\Delta_{0}=\operatorname{Re}\left(M_{\Delta}\right)-M$. Fig. 2 shows the $\Delta$-contributions up to the NLO. At this order, the spin-polarizabilities are still parameter-free predictions. However, the large paramagnetic $\Delta$-effects and the included pion contributions are not sufficient to explain the observed proton magnetic polarizability. This necessitates the introduction of two lowenergy coefficients $\delta \alpha, \delta \beta$. These "off-sets" are then determined from data. It should be mentioned here that the $\Delta$-isobar has been included in the $\gamma \mathrm{d}$ calculation only. 
$19^{\text {th }}$ International IUPAP Conference on Few-Body Problems in Physics

\section{The nucleon inside a nucleus}

The $\gamma$-nucleus scattering amplitude is written as

$$
\mathcal{M}=\left\langle\Psi_{f}|\hat{O}| \Psi_{i}\right\rangle
$$

with $\left|\Psi_{i}\right\rangle$ and $\left|\Psi_{f}\right\rangle$ being the nuclear wavefunctions. $\hat{O}$ represents the photon-nucleon(s) interaction kernel that is calculated using $\chi \mathrm{PT}$. The nuclear wavefunctions are usually derived from a potential model or a chiral potential.

Neutron properties are usually extracted from data taken on few-nucleon systems by dis-entangling nuclear-binding effects. Within the power-counting scheme used for these calculations, these effects include pion-exchange mechanisms between two nucleons at the lowest order (see Fig. 3). The external photon may either couple to a pion-nucleon vertex or an exchanged pion. The graphs shown in Fig. 3 enter the $\gamma \mathrm{d}$ or $\gamma^{3} \mathrm{He}$ calculation only at next-to-leading order $\left(O\left(Q^{3}\right)\right)$. Additional contributions at NNLO have also

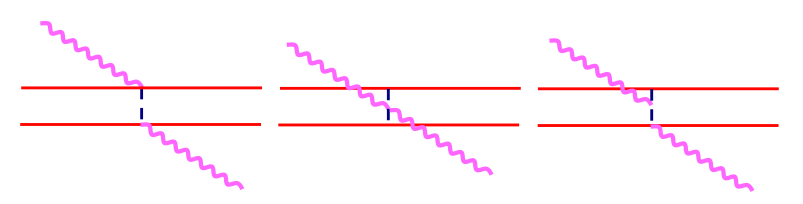

Fig. 3. Lowest-order two-body current contributions. They enter a $\gamma$-nucleus calculation at NLO. Crossed graphs and graphs with the nucleons interchanged are not shown.

been calculated [16] but are not essential for this discussion.

\section{Observables}

The availability of polarized beams and the technology to polarize targets has opened up the field of possible measurements. Over and above the canonical differential crosssection, one can now measure observables that include different combinations of unpolarized/polarized beam and target. Fig. 4 gives a representation of the observables considered. The convention adopted is that the beam direction is the $z$-axis and the $x-z$ plane is the scattering plane. For a linearly polarized beam and unpolarized target, $\left.\frac{\mathrm{d} \sigma}{\mathrm{d} \Omega}\right|_{x} ^{\text {lin }}$ is the differential cross-section for photon polarization in the scattering plane, and $\left.\frac{\mathrm{d} \sigma}{\mathrm{d} \Omega}\right|_{y} ^{\text {lin }}$ for perpendicular polarization. The $\Delta \mathrm{s}$ are the double polarized observables that involve a vector-polarized deuteron and a circularly or linearly polarized photon. $\Delta_{x(z)}^{\text {circ }}$ gives the difference in the difference in the differential cross-sections between configurations when the target is polarized along $+\hat{x}(+\hat{z})$ and $-\hat{x}(-\hat{z})$ using a circularly polarized beam. $\Delta_{x(z)}^{\operatorname{lin}}$ gives the photon polarization asymmetry when the target is polarized along $+\hat{x}(+\hat{z})$. Experimental measurents often use the asymmetries $\Sigma$ obtained by dividing the $\Delta \mathrm{s}$ by the sum of the measured differential cross-sections. Of course, these asymmetries are then devoid of systematic uncertainties.

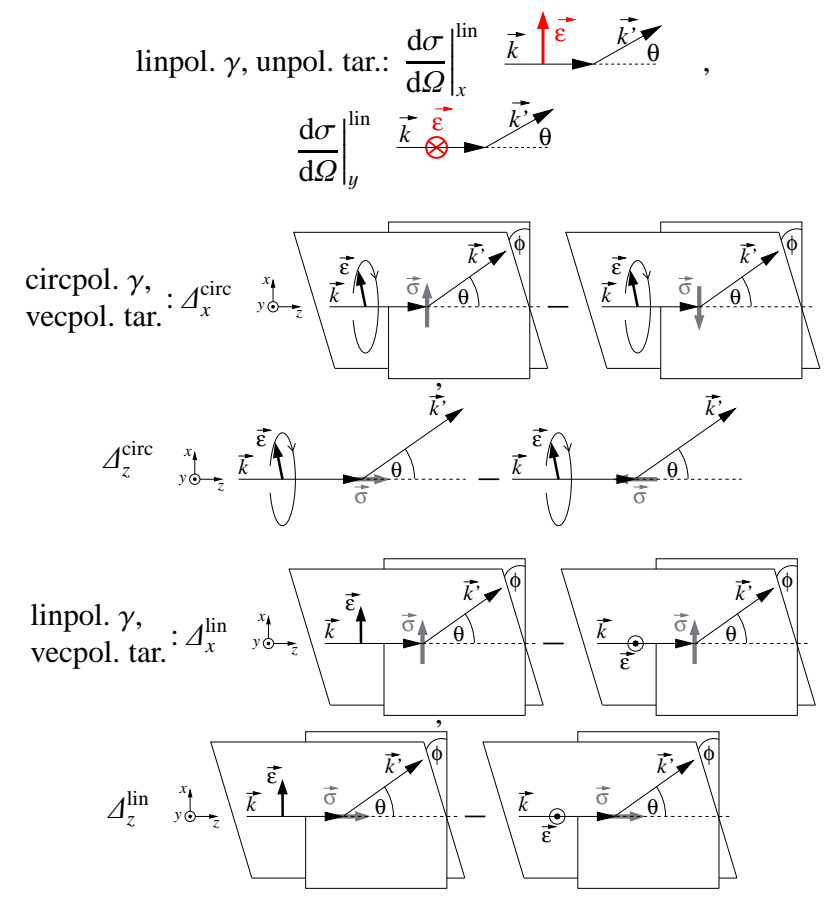

Fig. 4. Definition of observables for singly and double polarized observables. Figure from H. Grießhammer.

The observables described above are only a subset of all the possible combinations. The focus of these proceedings is only on some prominent examples. For the deuteron, representative results for most of the above obervables are presented. The aim is to aid in planning new experiments, and the results for all of the above observables are available as an interactive Mathematica 7.0 notebook from Grießhammer (hgrie@gwu.edu).For the ${ }^{3} \mathrm{He}$ Compton scattering calculation results for the unpolarized differential cross-section and $\Delta_{x(z)}^{\text {circ }}$ only are presented.

\section{Elastic $\gamma \mathbf{d}$ scattering}

The NLO $\left(O\left(\varepsilon^{3}\right)\right)$ deuteron Compton scattering calculations in the SSE variant of $\chi \mathrm{PT}$ include the amplitudes of Secs. 2 and 3. Note that the pion-pole graph of Fig. 1 does not contribute to deuteron Compton scattering as deuteron is an isoscalar. There is an additional ingredient in the calculation - resumming the intermediate $N N$-rescattering states. A consistent description of $\gamma \mathrm{d}$ Compton scattering must also give the correct Thomson limit, an exact lowenergy theorem which in turn follows from gauge invariance [23]. In deuteron Compton scattering, this mandates the inclusion of $T_{N N}$ whenever both nucleons propagate close to their mass-shell between photon absorption and emission, i.e. when the photon energy $\omega \lesssim 50 \mathrm{MeV}$. At higher photon energies $\omega \gtrsim 60 \mathrm{MeV}$, the nucleon is kicked far enough off its mass-shell, $E \sim Q$, for the amplitude to become perturbative. Figure 5 lists the additional contributions to Compton scattering off the deuteron to next-toleading order in $\chi \mathrm{EFT}$ when the low-energy resummation of the intermediate $N N$-rescattering states is performed. 


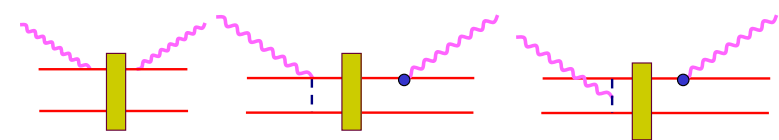

Fig. 5. Low-energy $N N$ rescattering contributions to deuteron Compton scattering. The rectangle in the middle represents the $N N$ T-matrix.

Once all the ingredients in $\hat{O}$ of Eq. (9) are determined, it is folded with deuteron wavefunctions to obtain the deuteron Compton amplitude, using which any observable can be calculated. For the results reported here we use chiral NNLO wavefunctions for the deuteron and the $A V 18$ potential in the intermediate $N N$-rescattering process. While it is desirable that for a consistent calculation the $\gamma N N$ kernel, the wavefunctions and the potential used to calculate the intermediate rescattering contribution should be derived using the same framework. Our $\gamma N N$ kernel and the wavefunctions are indeed derived from the same framework, but $A V 18$ is not a chiral potential. However, it has been shown in Ref. [4] and since then we have verified that the form of the potential used in the intermediate rescattering process causes barely perceivable difference in the final results. Issues of matching currents and couplings etc. only appear at two higher orders than what we calculate.

Also note that the polarizabilities extracted from deuteron Compton scattering are the isoscalar combinations. This means that the extraction of the neutron polarizabilities will depend on how accurately the proton polarizabilities are known.

\subsection{Significance of the $\triangle$ and $N N$ Rescattering on Polarization Observables}

We first analyse the impact of including the $\Delta$-isobar and intermediate $N N$ rescattering contributions on polarization observables. Figure 6 compares double-polarization observables within different schemes. The top row shows $\Delta_{z}^{\text {circ }}$ (left: $45 \mathrm{MeV}$ and right: $125 \mathrm{MeV}$ ) and the bottom row shows $\Delta_{x}^{\text {circ }}$ (left: $45 \mathrm{MeV}$ and right: $125 \mathrm{MeV}$ ). In the lefthand panels, the dashed line is a $O\left(Q^{3}\right) \mathrm{HB} \chi \mathrm{PT}$ calculation without dynamical $\Delta$ or rescattering, the dot-dashed line is the same calculation with $N N$-rescattering included, and the solid line is a $O\left(\varepsilon^{3}\right)$ calculation with both intermediate $N N$ rescattering and a dynamical $\Delta$. In the right-hand panels, the dashed line is a $O\left(Q^{3}\right) \mathrm{HB} \chi \mathrm{PT}$ calculation without dynamical $\Delta$, the dot-dashed line has the dynamical $\triangle$ added, but no $N N$-rescattering, and the solid line is the full calculation with intermediate $N N$ rescattering and a dynamical $\triangle$ As for unpolarized observables [5,19], the $\Delta(1232)$ does not contribute appreciably at $45 \mathrm{MeV}$, but the observable is still ruled by including intermediate $N N$ rescattering for the correct Thomson limit. In contrast, the $\Delta$ and intermediate $N N$-rescattering are equally significant at $125 \mathrm{MeV}$.

Thus, together with the observations of Refs. [5, 19] for unpolarized deuteron Compton scattering, we emphasize that the $\Delta$-isobar and the intermediate $N N$ - rescattering contributions are necessary ingredients of our calculations that attempt to identify observables for the extraction of neutron polarizabilities in the energy range $45-125 \mathrm{MeV}$ (lab). It has been verified that the dependence on the potential that is used to generate the deuteron wavefunctions and the $N N$ T-matrix (in the intermediate state) is negligible.

\subsection{Results}
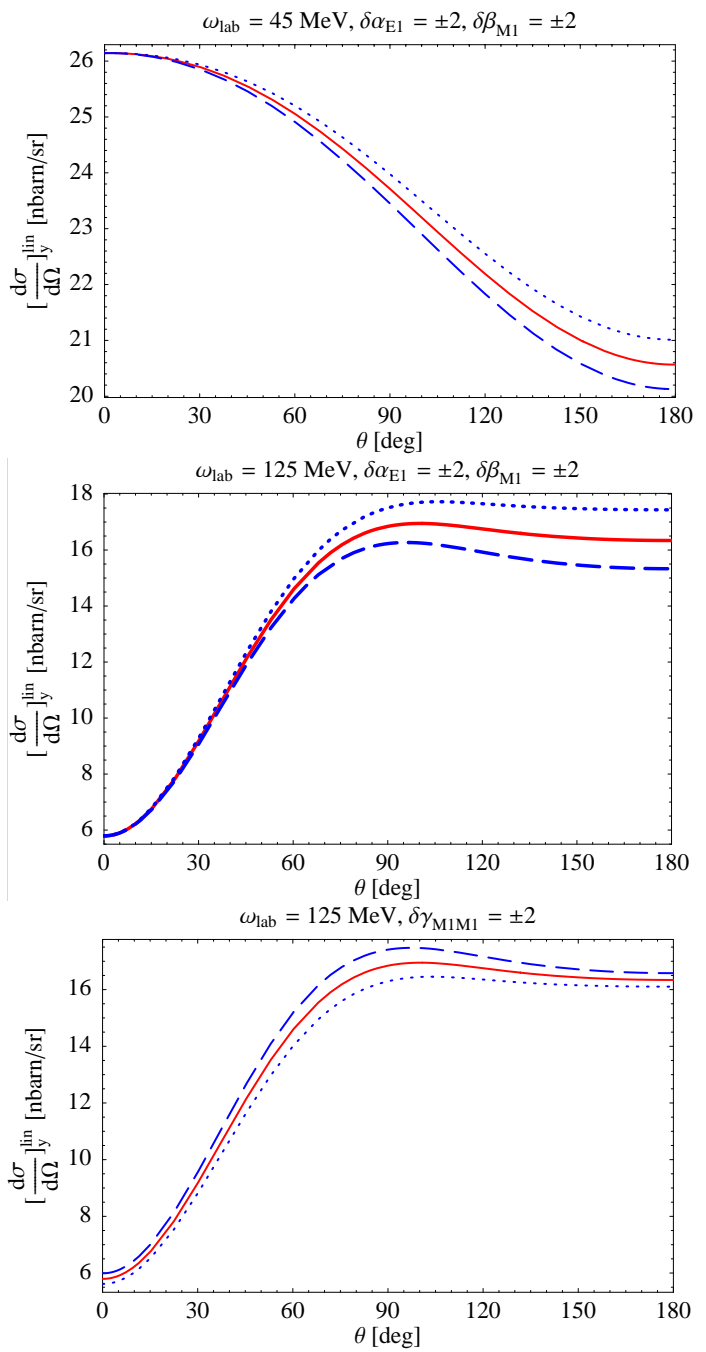

Fig. 7. Differential cross-sections with photons linearlypolarized along the $y$-axis. Top and middle panels: The combination $\alpha^{E 1}-\beta^{M 1}$ is varied, while their sum is constrained by the Baldin sum rule. Bottom panel: $\gamma_{M 1 M 1}$ varied by \pm 2 units at $\omega_{\text {lab }}=125 \mathrm{MeV}$.

We now present results for selected polarization observables which show appreciable sensitivity to the polarizabilities. Strictly speaking, at this order only $\alpha_{E 1}$ and $\beta_{M 1}$ have an undertermined short-distance piece; the spinpolarizailities are predictions of the theory. However, we 

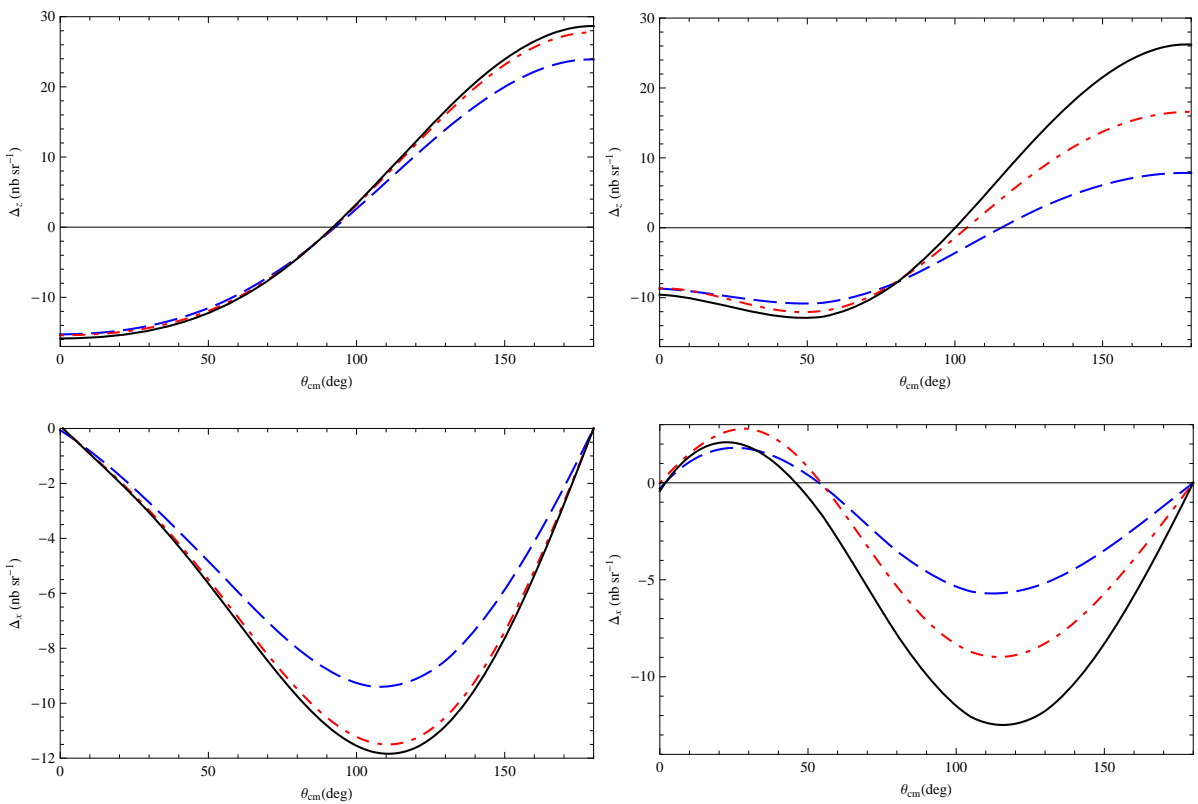

Fig. 6. Effects of $\Delta(1232)$ and of resumming $N N$ intermediate states on the double polarization observables $\Delta_{z}$ (top) and $\Delta_{x}$ (bottom). Left: $\omega_{\text {lab }}=45 \mathrm{MeV}$; right: $\omega_{\text {lab }}=125 \mathrm{MeV}$. Legend given in the text. 2

have adopted the strategy whereby we arbitrarily add six parameters $\delta \alpha_{E 1}, \delta \beta_{M 1}$ and $\delta \gamma_{i j}$ s to the calculation to represent effects not explictly included in the theory. This allows us to gauge the dependence of the observables on these parameters.

The topmost panel of Fig. 7 shows $\left.\frac{\mathrm{d} \sigma}{\mathrm{d} \Omega}\right|_{y} ^{l i n}$ at $45 \mathrm{MeV}$ (lab). At this energy, there is appreciable sensitive only to $\alpha_{E 1}$ and $\beta_{M 1}$. At $125 \mathrm{MeV}$ (lab) (middle and bottom panels) however, the sensitivity to $\gamma_{M 1 M 1}$ is large and comparable to that of $\alpha_{E 1}-\beta_{M 1}$. Note that the sensitivity to $\gamma_{M 1 M 1}$ decreases at back angles where the sensitivity to $\alpha_{E 1}-\beta_{M 1}$ is largest. Fig. 8 shows the double-polarization observable $\Delta_{z}^{\text {lin }}$ at $\omega_{\text {lab }}=125 \mathrm{MeV}$. There is comparable sensitivity to three of the polarizabilities $\alpha_{E 1}, \beta_{M 1}$ and $\gamma_{M 1 M 1}$. Finally, we show the double-polarization observable $\Delta_{x}^{\text {circ }}$ at $\omega_{\mathrm{lab}}=$ $125 \mathrm{MeV}$ in Fig. 9. There is appreciable and comparable sensitivity to $\alpha_{E 1}, \beta_{M 1}$ and $\gamma_{E 1 E 1}$, but only minor sensitivity to the other spin-polarizabilities.

The other observables (not shown here) also show minor sensitivity to some of the polarizabilities. The message to take away from this analysis is that no obervable is sensitive to only one of the polarizabilities. Ideally, the extraction of the six polarizabilities should be performed from a global fit to a number of experimental measurements. Practically however, one should be judicious in selecting suitable observables for the extraction the polarizabilties. Configurations can be isolated where the contribution of one (or more) of the polarizabilities is zero. For example, see Fig. 10 where a detector under $90^{\circ}$ can therefore not detect $M 1$ photons radiated from the induced magnetic dipole in the nucleon, cf. [24].

Also note that the sensitivity to $\alpha_{E 1}$ and $\beta_{M 1}$ manifests at much lower energies (see for e.g. Fig. 7 and 10) compared to the spin polarizabilities. It is therefore advisable to focus on $\alpha_{E 1}$ and $\beta_{M 1}$ at lower energies and the spin polarizabilities at higher energies. It is imperative that the values of the electric and magnetic polarizabilities be better extracted so as not to taint any extraction of the spin polarizabilities.

\section{Elastic $\gamma^{3}$ He scattering}

This section reports calculations for ${ }^{3} \mathrm{He}$ Compton scattering at $O\left(Q^{3}\right)$ in $\mathrm{HB} \chi \mathrm{PT}$. The operator $\hat{O}$ in Eq. (9) is the irreducible amplitude for elastic scattering of real photons from the NNN system. At $O\left(Q^{3}\right)$ this operator encodes the physics of two photons coupling to a two-nucleon system inside the ${ }^{3} \mathrm{He}$ nucleus. We do not have to include any irreducible three-body Compton mechanisms in our calculation because they appear at the earliest at $O\left(Q^{5}\right)$. This allows us to treat the ${ }^{3} \mathrm{He}$ nucleus as a $(2+1)$ nucleon system and enables the simplification of Eq. (9) to:

$$
\mathcal{M}=3\left\langle\Psi_{f}\left|\frac{1}{2}\left(\hat{O}^{1 B}(1)+\hat{O}^{1 B}(2)\right)+\hat{O}^{2 B}(1,2)\right| \Psi_{i}\right\rangle
$$

using the Faddeev decomposition of $|\Psi\rangle$. The structure of the calculation is then similar for the one- and two-body parts. The superscript $1 B$ in $\hat{O}^{1 B}(a)$ of Eq. (10) refers to the one-body mechanisms or the $\gamma \mathrm{N}$ amplitude where the external photon interacts with nucleon ' $a$ ' (refer to Fig. 1). Similarly, $\hat{O}^{2 B}(a, b)$ represents two-body mechanisms where the external photons interact with the pair ' $(a, b)$ ' (refer to Fig. 3).

We calculate $\mathcal{M}$ on a partial-wave Jacobi basis. Convergence of the results with respect to the angular-momentum expansion was confirmed. For details on the calculational procedure see Ref. [12-14]. These ${ }^{3} \mathrm{He}$ Compton 

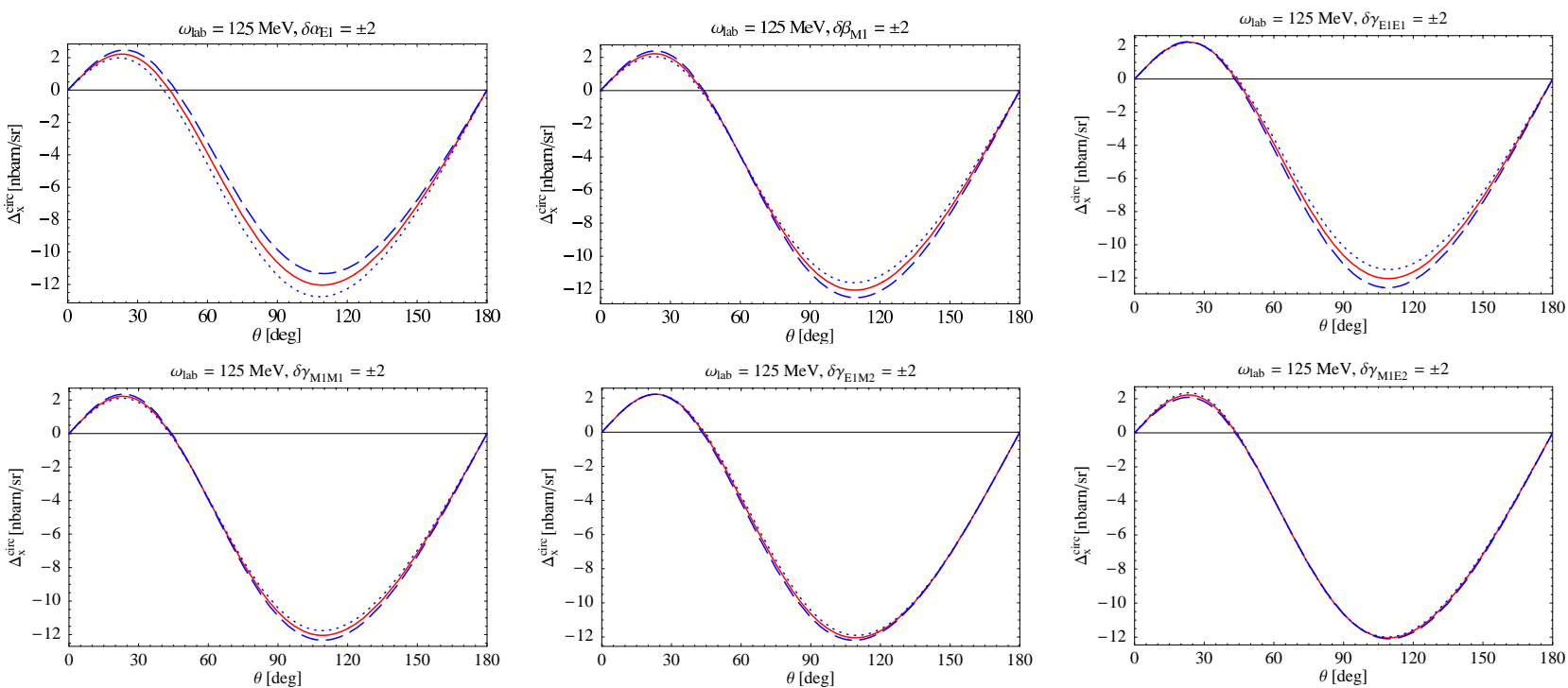

Fig. 9. The double-polarization asymmetry $\Delta_{z}^{\text {circ }}$ with circularly-polarized photons at $\omega_{\text {lab }}=125 \mathrm{MeV}$. From top left to bottom right, variation by \pm 2 units of $\alpha_{E 1}, \beta_{M 1}, \gamma_{E 1 E 1}, \gamma_{M 1 M 1}, \gamma_{E 1 M 2}, \gamma_{M 1 E 2}$.

scattering calculations are the first such calculations. In future, we intend to extend these calculations to include: the next higher order interactions, the $\Delta$-isobar as an explicit degree of freedom and also the effects of intermediate $N N N$-rescattering.

\subsection{Results}

The amplitude (10) is now used to calculate observables. In Fig. 11 we plot our $O\left(Q^{3}\right)$ differential cross-section predictions for elastic $\gamma^{3} \mathrm{He}$ scattering. The two panels are for $\omega=60$ and $120 \mathrm{MeV}$. Both show three different dcs calculations- $O\left(Q^{2}\right)$ :dashed curve, IA (Impulse Approximation): dot-dashed curve and $O\left(Q^{3}\right)$ : solid curve. The $O\left(Q^{2}\right)$ calculation includes only the proton Thomson term, since that is the LO $\gamma \mathrm{N}$ amplitude in $\chi \mathrm{PT}$ at that order. The IA calculation contains only one-body $O\left(Q^{3}\right)$ contribution (graphs in Fig. 1. There is a sizeable difference between the $I A$ and the $O\left(Q^{3}\right)$ confirming that two-body currents are equally important. Also, the difference between $O\left(Q^{3}\right)$ and $O\left(Q^{2}\right)$ is very small at $60 \mathrm{MeV}$ - showing that $\chi \mathrm{PT}$ may converge well there. This difference gradually increases with energy which may be partly because the fractional effect of the polarizabilities increases with $\omega$.

To analyze the effect of $\alpha$ and $\beta$ on the dcs, in Fig. 12 we plot the $O\left(Q^{3}\right)$ des at $80 \mathrm{MeV}$ obtained when we add shifts, $\Delta \alpha^{(n)}$ and $\Delta \beta^{(n)}$, to the $O\left(Q^{3}\right)$ values of $\alpha$ and $\beta$ for the neutron (see for e.g Ref. [2]). $\Delta \alpha^{(n)}$ is varied in the range $(-4 \ldots 4) \times 10^{-4} \mathrm{fm}^{3}$ (long-dashed curve ... dashed curve) in steps of $2 \times 10^{-4} \mathrm{fm}^{3}$. Similarly, $\Delta \beta^{(n)}$ between $(-2 \ldots 6) \times 10^{-4} \mathrm{fm}^{3}$ (long-dashed curve ... dashed curve) in steps of $2 \times 10^{-4} \mathrm{fm}^{3}$. This assesses the impact that one set of higher-order mechanisms has on our $O\left(Q^{3}\right)$ predictions. Notice that the sensitivity to $\beta^{(n)}$ vanishes at $\theta=90^{\circ}$. This is because $\alpha^{(n)}$ and $\beta^{(n)}$ enter $A_{1}^{(n)}$ in the combination $\alpha^{(n)}+\beta^{(n)} \cos \theta$. This implies that $\alpha^{(n)}$ and $\beta^{(n)}$ can be extracted independently from the same experiment. Secondly, the absolute size of the shift in the dcs due to $\Delta \alpha^{(n)}$ and $\Delta \beta^{(n)}$ is roughly the same for all energies. This suggests that measurements could be done at $\omega \approx 80 \mathrm{MeV}$, where the count rate is higher, and the contribution of higherorder terms in the chiral expansion should be smaller. Thirdly, the sequence of the curves for different values of $\Delta \beta^{(n)}$ reverses if one compares the forward and backward angles. This suggests that a meaurement of the forward to back angle ratio of the dcs will enhance the effect of $\Delta \beta^{(n)}$.

Before examining double-polarization observables in $\gamma^{3} \mathrm{He}$ scattering we try to develop some intuition for the $\gamma^{3} \mathrm{He}$ amplitude. ${ }^{3} \mathrm{He}$ is a spin- $\frac{1}{2}$ target - this means that the matrix element (10) can be decomposed in the same fashion as the nucleon's Compton matrix element in Eq. (3).

$$
T_{\gamma^{3} H e}=\sum_{i=1 \ldots 6} A_{i}^{3} H e(\omega, \theta) t_{i} ; \quad A_{i}^{3} H e=A_{i}^{1 B}+A_{i}^{2 B},
$$

where $A_{i}^{1 B}\left(A_{i}^{2 B}\right)$ comes from considering the matrix element of the one-body (two-body) operators in Eq. (10). The structures $t_{3}-t_{6}$ now involve the 'nuclear' spin. In the ground state of polarized ${ }^{3} \mathrm{He}$, the two proton spins are anti-aligned for the most part, therefore the nuclear spin is largely carried by the unpaired neutron [11]. We find that the $O\left(Q^{3}\right)$ two-body currents $A_{1}^{2 B}$ and $A_{2}^{2 B}$ are numerically sizeable, but $A_{3}^{2 B}-A_{6}^{2 B}$ are negligible. Hence, to the extent that polarized ${ }^{3} \mathrm{He}$ is an 'effective neutron', we expect $A_{i}^{3} H e=A_{i}^{(n)}$ for $i=3-6$. Thus, it is possible to look for the effects of the 'neutron' spin polarizabilities directly in a polarized ${ }^{3} \mathrm{He}$ Compton scatteing experiment. Moreover, since $A_{1}^{3} \mathrm{He}$ is dominated by the two proton Thomson terms, we anticipate a more enhanced signal from the neutron spin polarizabilities than is predicted for the corresponding $\gamma$ d observables (see Sec. 5.2 and Refs. [20,25]).

We emphasize that these arguments are meant only as a guide to the physics of our exact $O\left(Q^{3}\right)$ calculation. Our 
$19^{\text {th }}$ International IUPAP Conference on Few-Body Problems in Physics
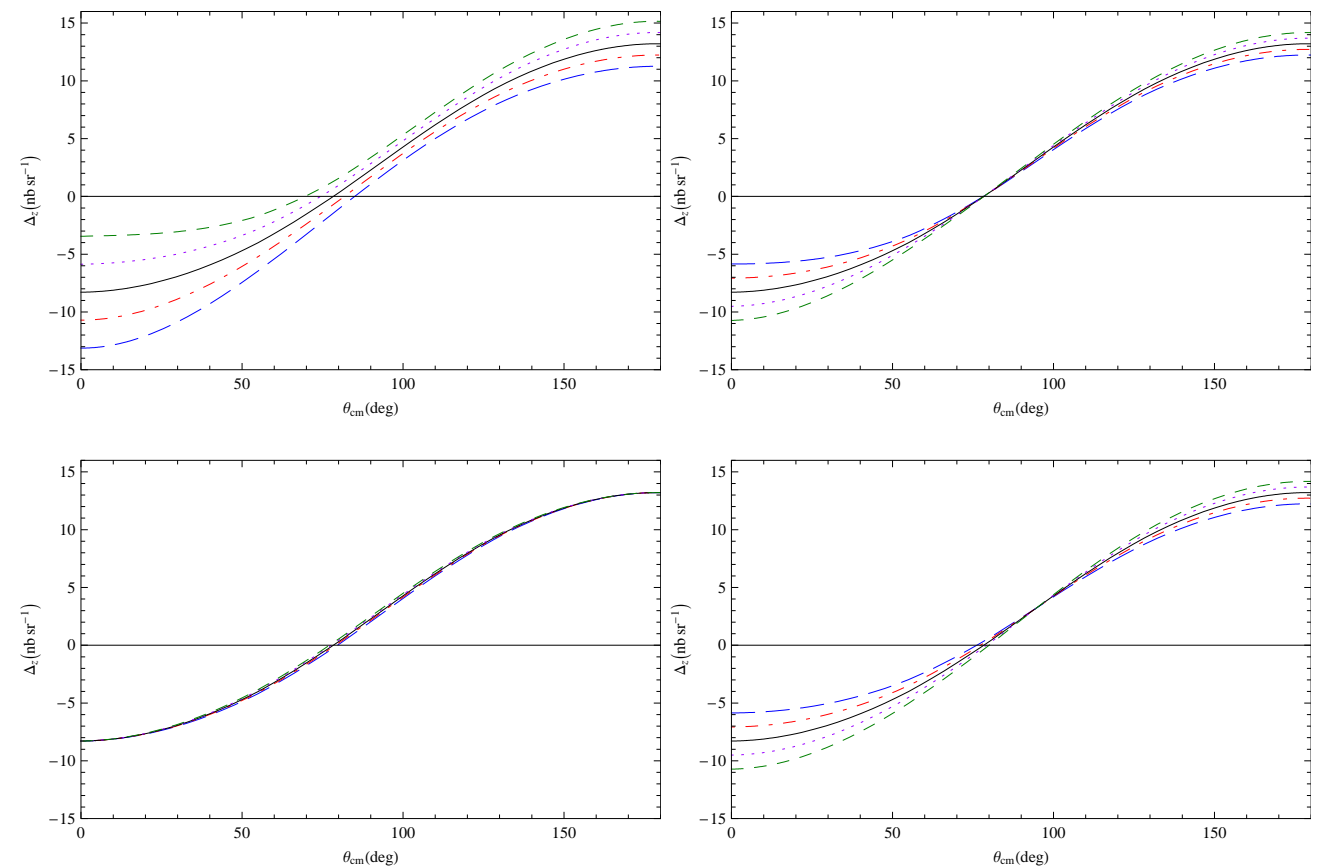

Fig. 13. $\Delta_{z}^{\text {circ }}$ at $\omega_{\mathrm{cm}}=120 \mathrm{MeV}$ with $\gamma_{1}^{(n)}$ (top left) $-\gamma_{4}^{(n)}$ (bottom right) varied one at a time. The spin polarizabilities are varied by $\pm 100 \%$ of their $O\left(Q^{3}\right)$ values [2].
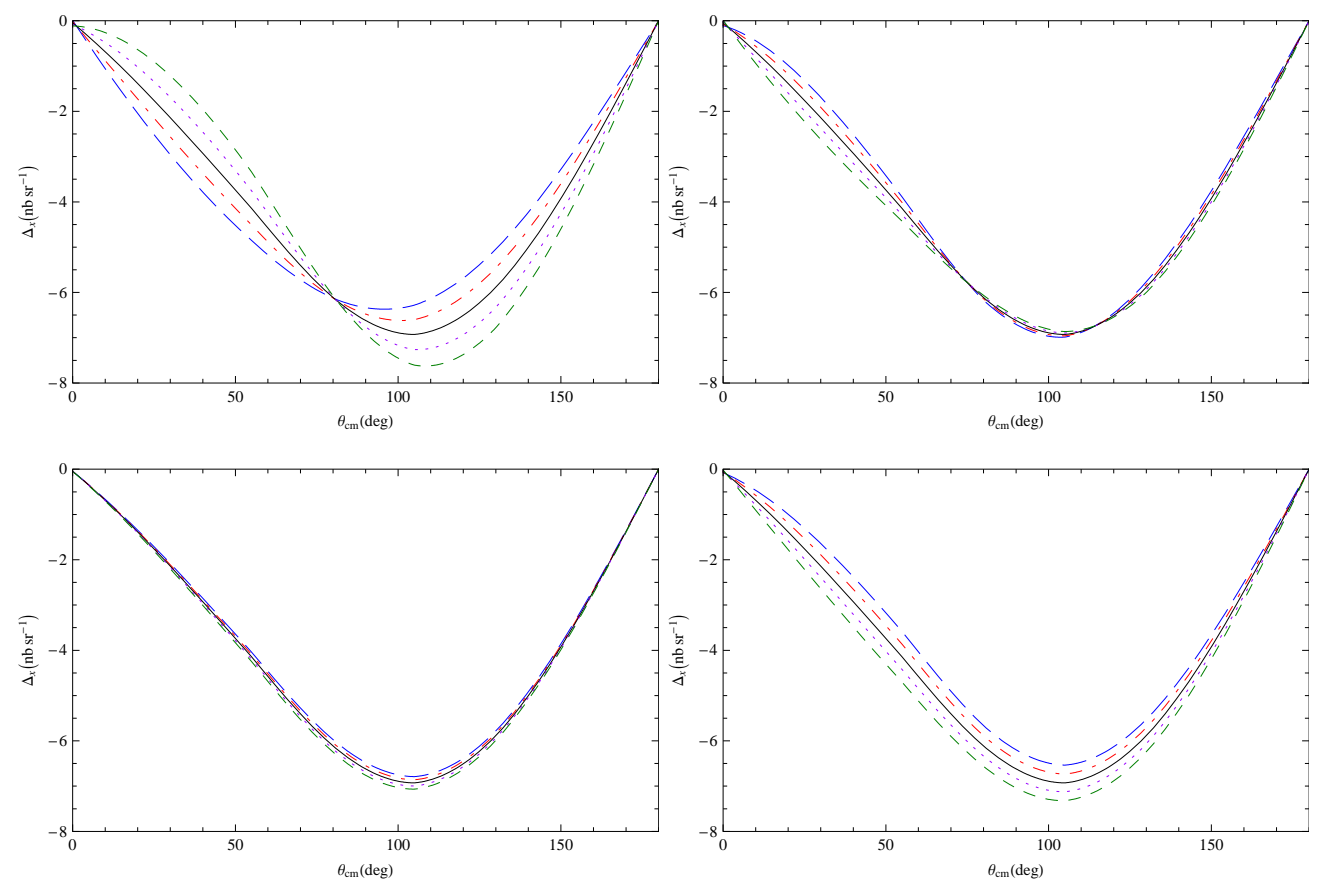

Fig. 14. $\Delta_{x}^{\text {circ }}$ (c.m. frame) at $\omega_{\mathrm{cm}}=120 \mathrm{MeV}$ when $\gamma_{1}^{(n)}$ (top left) $-\gamma_{4}^{(n)}$ (bottom right) are varied one at a time. The spin polarizabilities are varied by $\pm 100 \%$ of their $O\left(Q^{3}\right)$ values [2].

${ }^{3} \mathrm{He}$ wave function is obtained by solving the Faddeev equations with $\mathrm{NN}$ and $3 \mathrm{~N}$ potentials derived from $\chi \mathrm{PT}$. All of the effects due to neutron depolarization and the spin-dependent pieces of $\hat{O}^{2 B}$ are included in our calculation of the amplitude (9). This yields the results for $\Delta_{z}^{\text {circ }}$ and $\Delta_{x}^{\text {circ }}$ shown in Figs. 13 and 14. In these figures we have chosen to vary $\gamma_{1}^{(n)}, \gamma_{2}^{(n)}$, and $\gamma_{2}^{(n)}$ and $\gamma_{4}^{(n)}$. (These are parameterizations of the spin polarizabilities following Ragusa [1]. $\gamma_{1}^{(n)}, \gamma_{2}^{(n)}$, and $\gamma_{2}^{(n)}$ and $\gamma_{4}^{(n)}$ are linear combi- 


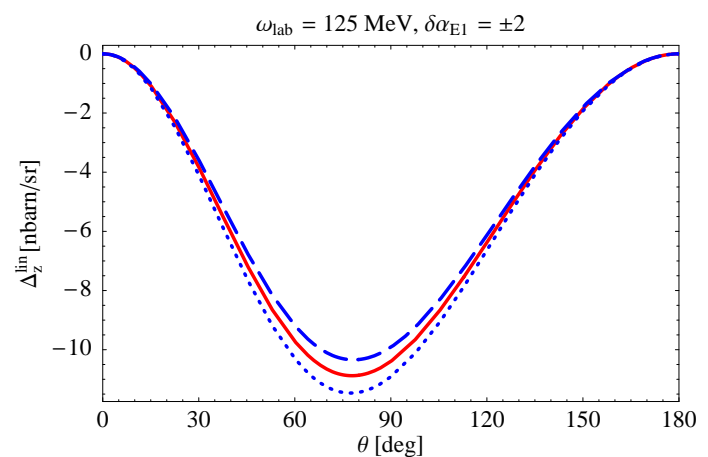

$\omega_{\text {lab }}=125 \mathrm{MeV}, \delta \beta_{\mathrm{M} 1}= \pm 2$
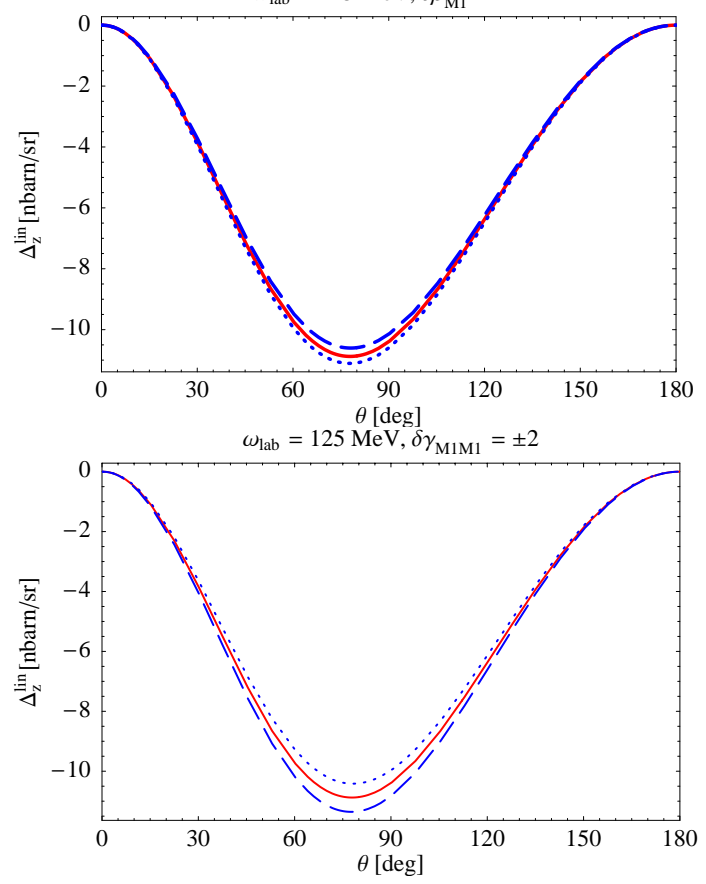

Fig. 8. The double-polarization observable $\Delta_{z}^{\text {lin }}$ with linearlypolarized photons at $\omega_{\mathrm{lab}}=125 \mathrm{MeV} . \alpha^{E 1}\left(\beta_{M 1}\right)$ is varied by \pm 2 units in the topmost (middle) panel. The bottom panel shows variation of $\gamma_{M 1 M 1}$ by \pm 2 .

nations of the multipole parameterizations of Eq. (2).) In both Fig. 13 and Fig. 14 the $\Delta \gamma_{1}^{(n)} \ldots \Delta \gamma_{4}^{(n)}$ (top left to bottom right) are varied one by one by $\pm 100 \%$ of their $O\left(Q^{3}\right)$ predicted values [2]. Both $\Delta_{z}^{\text {circ }}$ and $\Delta_{x}^{\text {circ }}$ are quite sensitive to $\gamma_{1}^{(n)}, \gamma_{2}^{(n)}$, and $\gamma_{4}^{(n)}-\Delta_{z}^{\text {circ }}$ seems to be sensitive to the combination $\gamma_{1}^{(n)}-\left(\gamma_{2}^{(n)}+2 \gamma_{4}^{(n)}\right) \cos \theta$ whereas $\Delta_{x}^{\text {circ }}$ is sensitive to a different one. With the expected photon flux at an upgraded $\mathrm{HI} \gamma \mathrm{S}$ such effects can be measured [26]. Notice that two different linear combinations of $\gamma_{1}^{(n)}, \gamma_{2}^{(n)}$, and $\gamma_{4}^{(n)}$ can be extracted through measurements at different angles. For a more detailed discussion see $[12,14]$. Thus, $\Delta_{z}^{\text {circ }}$ and $\Delta_{x}^{\text {circ }}$ are sensitive to two different linear combinations of $\gamma_{1}^{(n)}, \gamma_{2}^{(n)}$, and $\gamma_{4}^{(n)}$ and their measurement should provide an unambiguous extraction of $\gamma_{1}^{(n)}$, as well as constraints on $\gamma_{2}^{(n)}$ and $\gamma_{4}^{(n)}$.
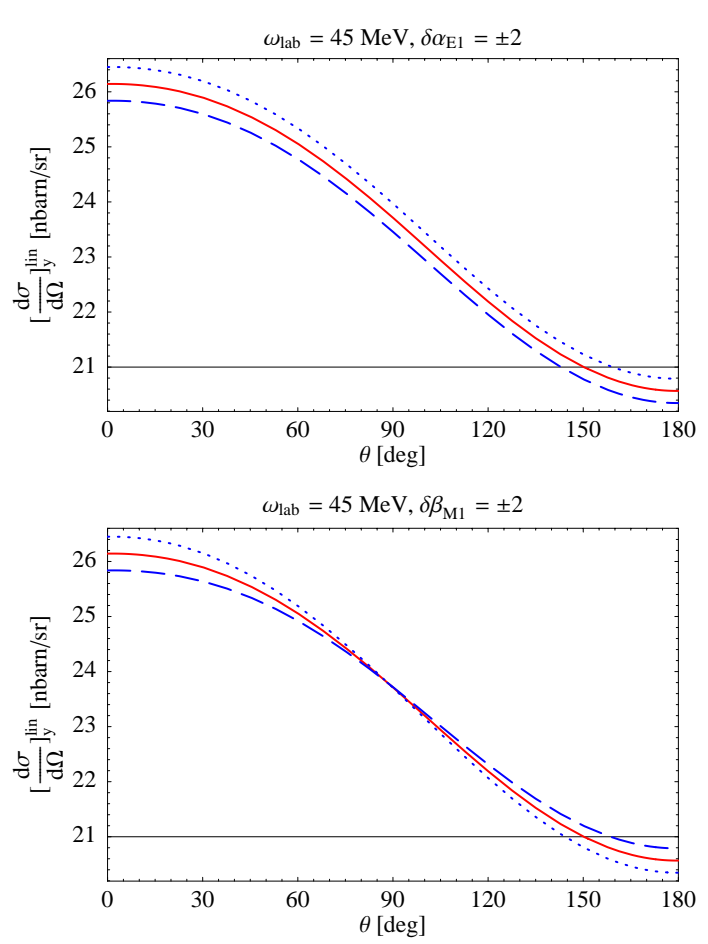

Fig. 10. Plots of $\left[\frac{d \sigma}{d \Omega}\right]_{y}^{\text {in }}$ at $\omega_{\text {lab }}=45 \mathrm{MeV}$ with varying $\alpha_{E 1}$ (top) and $\beta_{M 1}$ (bottom).Notice that the sensitivity to $\beta_{M 1}$ vanishes at $90 \mathrm{deg}$.

\section{Summary and Outlook}

Results of elastic deuteron and ${ }^{3} \mathrm{He}$ Compton scattering have been presented with the aim of extracting neutron polarizabilities. Our results show that most of the observables are quite sensitive to the polarizabilities. The electric and magnetic polarizabilities can also be directly extracted from the unpolarized deuteron Compton scattering dcs (as shown in Refs. $[4,5,16])$ and unpolarized dcs measurements are underway at MAXLab in Sweden [10]. It is essential to accurately know the values of the electric and magnetic polarizabilities before focussing on the spin polarizabilities which are 'higher-order' effects. The spin polarizabilities start playing a role at energies of around 80 $90 \mathrm{MeV}$. Our results have shown that some of the doublepolarization observables are sensitive to different linear combinations of the spin polarizabilities.

In view of our results we would like to advocate that-

1. Since accurate extraction of the electric and magnetic polarizabilities is central to the plan of extracting the six polarizabilities, a number of relatively low-energy experiments $(\lesssim 80 \mathrm{MeV})$ should focus on extracting $\alpha^{(n)}$ and $\beta^{(n)}$. Apart from the aforementioned unpolarized deuteron Compton scattering experiment at MAXLab, an experiment at $\mathrm{HI} \gamma \mathrm{S}$ has also been approved. Planning is also underway for a ${ }^{3} \mathrm{He}$ Compton scattering experiment at $\mathrm{HI} \gamma \mathrm{S}$ [26]. Using two different nuclei for these measurements would help us better understand the nuclear effects. Also note that some of the single/double-polarization observables can also be 
$19^{\text {th }}$ International IUPAP Conference on Few-Body Problems in Physics
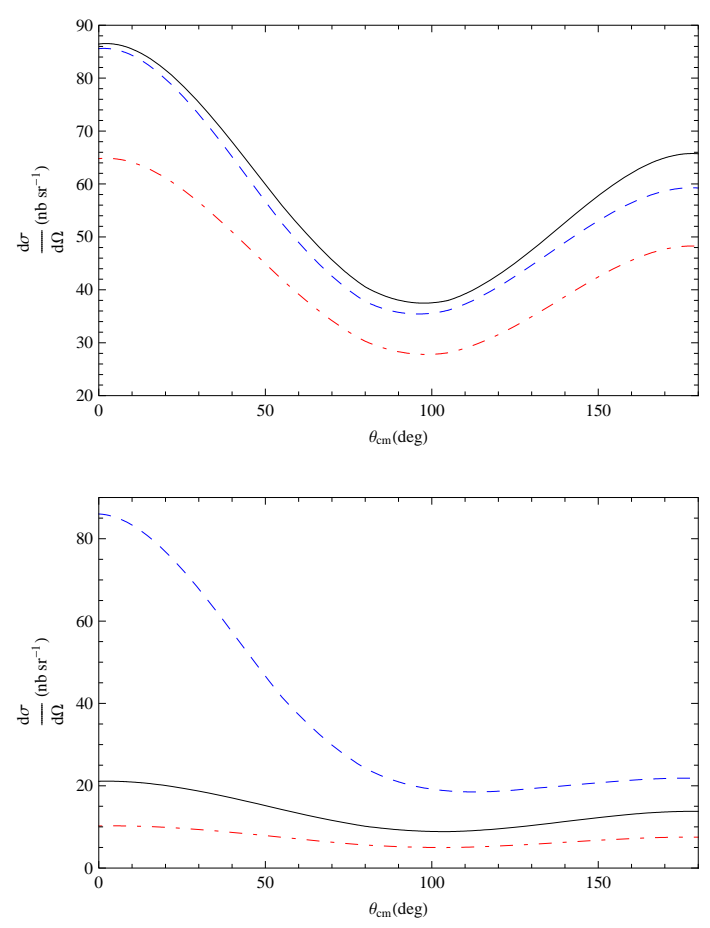

Fig. 11. Comparison of different c.m.-frame dcs calculations at $\omega_{\mathrm{cm}}=60 \mathrm{MeV}$ (top panel) and $\omega_{\mathrm{cm}}=120 \mathrm{MeV}$ (bottom panel). The dashed curve is the $O\left(Q^{2}\right)$ result, dot-dashed is the IA result and the solid curve is the $O\left(Q^{3}\right)$ result.
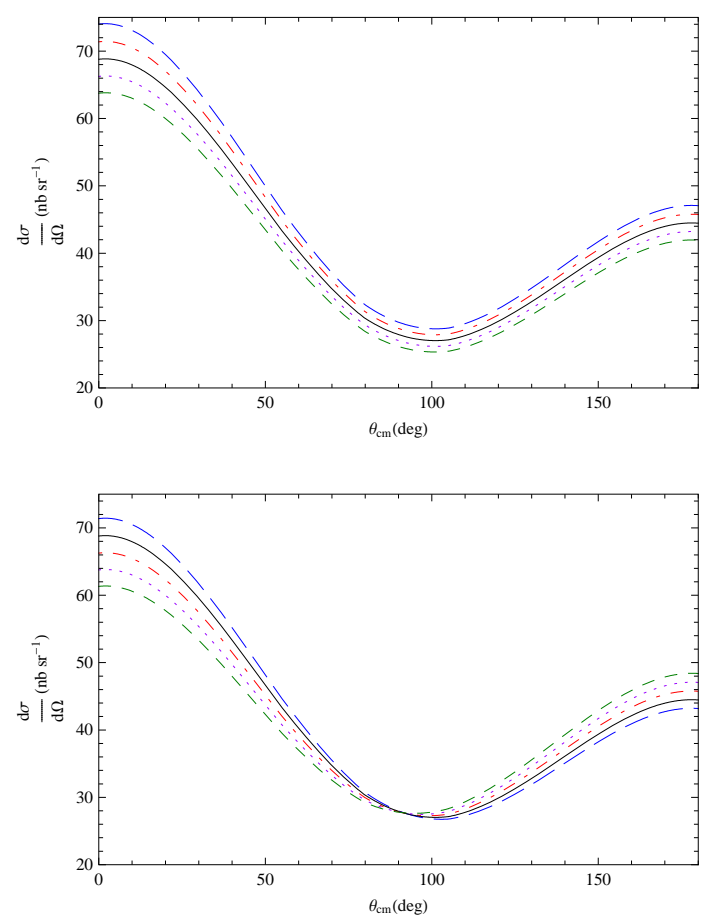

Fig. 12. The c.m.-frame $O\left(Q^{3}\right)$ dcs at $\omega_{\mathrm{cm}}=80 \mathrm{MeV}$ with varying $\Delta \alpha^{(n)}$ (left panel) and $\Delta \beta^{(n)}$ (right panel). used for these extractions. We emphasise that should such measurements be necessary, they should be done at lower energies where the effects of the spin polarizabilities are negligible.

2. Once $\alpha$ and $\beta$ are better known, a series of simultaneous polarized measurements at higher energies $(\gtrsim 100$ $\mathrm{MeV}$ but below the pion-production threshold) would be required to determine the spin polarizabilities. The best observables to hunt for the spin polarizabilities seem to be the double-polarization observables for both deuteron and ${ }^{3} \mathrm{He}$ Compton scattering. Measurement of several linear combinations of these from a series of experiments can make it ultimately possible to decouple the four independent spin polarizabilities.

Ideally, a global fit to a sizeable database that includes polarized/unpolarized measurements from different targets, from low to high (but below the pion-production threshold) energies at various angles would result in an unambiguous extraction of the polarizabilities. At the other extreme, one can argue that six measurements are sufficient to determine the six polarizabilities. However, realistically speaking we need a judicious combination of different kinds of measurements. Other experimental avenues may include quasifree deuteron and He-3 Compton measurements.

In the meantime, theoretical efforts should include efforts to improve the accuracy of current calculations. As far as deuteron Compton scattering calculations are concerned effort is ongoing to perform a full NNLO calculation [15] with nucleons, pions and the Delta as effective degrees of freedom. These would then be the state-of-the-art systematic calculations describing the deuteron Compton scattering process from the Thomson limit to the pion production threshold. The next steps for ${ }^{3} \mathrm{He}$ Compton scattering would involve (in order of importance)-

- incorporating the $\Delta$-isobar as an explicit degree of freedom. As in deuteron Compton scatering we expect the $\Delta$ to play a significant role even at energies $\sim 100 \mathrm{MeV}$.

- Following this, effort needs to be directed towards restoration of the Thomson limit in ${ }^{3} \mathrm{He}$ Compton scattering. This would include resumming the intermediat $N N N$-rescattering states.

- A complete NNLO extension with nucleons, pions and the $\Delta$ as explicit degrees of freedom.

Lastly, one should remember that these calculations/measurements assume that the proton polarizabilities are accurately known. This is true for $\alpha^{(p)}$ and $\beta^{(p)}$, but the poton spin polarizabilities are not well-known. Thus, there must be efforts to extract these quantities before concentrating on the neutron spin polarizabilities. It is indeed encouraging that polarized proton Compton scattering [27] measurements are in the pipeline precisely for this reason.

\section{Acknowledgements}

I would like to thank my collaborators H. Grießhammer, J. McGovern, A. Nogga and D. Phillips. This work was supported by the National Science Foundation (CAREER grant PHY-0645498) and US-DOE (DE-FG02-97ER41019 
and DE-FG02-95ER-40907). Furthermore, I would like to thank the organizers for the generous financial support and an excellent conference.

\section{References}

1. S. Ragusa, Phys. Rev. D47, 3757 (1993); B. R. Holstein, D. Drechsel, B. Pasquini, M. Vanderhaeghen, Phys. Rev. C61, 034316 (2000).

2. V. Bernard, N. Kaiser, and Ulf-G. Meißner, Int. J. Mod. Phys. E4, 193 (1995).

3. M. Schumacher, Prog. Part. Nucl. Phys. 55, 567 (2005).

4. R. P. Hildebrandt, H. W. Grießhammer, T. R. Hemmert, nucl-th/0512063 (2005).

5. R. P. Hildebrandt, Ph. D. Thesis, arXiv.org:nucl-th/0512064.

6. K. Kossert, et al., Eur. Phys. J., A16, 259 (2003).

7. V. Pascalutsa, and D. R. Phillips, Phys. Rev. C68, 055205 (2003).

8. M. Gell-Mann, M. L. Goldberger and W. E. Thirring, Phys. Rev. 95, 1612 (1954).

9. A. M. Sandorfi, M. Khandaker, and C. S. Whisnant, Phys. Rev. D50, R6681 (1994).

10. G. Feldman, et al., Few Body Sys. 44, 325 (2008); L. Myers, Private communication.

11. B. Blankleider and R. M. Woloshyn, Phys. Rev., C29, 538 (1984); J. L. Friar et al., Phys. Rev., C42, 2310 (1990).

12. D. Choudhury, Ph.D. Thesis, Ohio University, 2006.

13. D. Choudhury, A. Nogga and D. R. Phillips, Phys. Rev. Lett. 98232303 (2007).

14. D. Shukla, A. Nogga and D. R. Phillips, Nucl. Phys. A819 98 (2009).

15. H. Grießhammer, J. McGovern, D. R. Phillips, D. Shukla, work in progress.

16. S. R. Beane, M. Malheiro, J. A. McGovern, D. R. Phillips, U. van Kolck, Phys. Lett B567, 200 (2003). Erratum-ibid: Phys. Lett. B607 320 (2005); S. R. Beane, M. Malheiro, J. A. McGovern, D. R. Phillips, U. van Kolck, Nucl. Phys. A747, 311-361 (2005).

17. R. P. Hildebrandt, H. W. Grießhammer, T. R. Hemmert, B. Pasquini, Eur.Phys.J. A20 293(2004).

18. S. R. Beane, M. Malheiro, D. R. Phillips, U. van Kolck, Nucl. Phys. A656, 367-399 (1999).

19. R. P. Hildebrandt, H. W. Grießhammer, T. R. Hemmert, D. R. Phillips, Nucl. Phys., A748, 573 (2005).

20. D. Choudhury, and D. R. Phillips, Phys. Rev., C71, 044002 (2005).

21. J. A. McGovern, Phys. Rev. C63, 064608 (2001).

22. T. R. Hemmert, B. R. Holstein and J. Kambor, Phys. Rev.D55, 5598 (1997); T. R. Hemmert, B. R. Holstein, J. Kambor and G. Knöchlein, Phys. Rev. D57(9), 5746 (1998).

23. J.L. Friar, Ann. of Phys. 95, 170 (1975); H. Arenhövel, Z. Phys. A297, 129 (1980); M. Weyrauch and H. Arenhövel, Nucl. Phys. A408, 425 (1983).

24. L. C. Maximon, Phys. Rev. C 39 (1989) 347.
25. H. Grießhammer and D. Shukla, arXiv:nucl-th/0910.0053; D. Shukla and H. Grießhammer, forthcoming.

26. H. Gao, Private Communication.

27. R. Miskimen, in Proceedings of the 5th International Workshop on Chiral Dynamics, Theory and Experiment, Durham, NC, 2006, published by World Scientific, Singapore; H. Weller et al., Prog. Part. Nucl. Phys., 62, 257 (2009). 